\title{
Methods to measure the cosmic-ray composition with the Auger Engineering Radio Array
}

\author{
Fabrizia Canfora $^{1, \star}$ for the Pierre Auger Collaboration ${ }^{2, \star \star}$ \\ ${ }^{1}$ Nikhef and Radboud University Nijmegen \\ ${ }^{2}$ Observatorio Pierre Auger, Av. San Martín Norte 304, 5613 Malargüe, Argentina
}

\begin{abstract}
The mass composition of ultra-high-energy cosmic rays plays a key role in the understanding of the origins of these rare particles. A composition-sensitive observable is the atmospheric depth at which the air shower reaches the maximum number of particles $\left(X_{\max }\right)$. The Auger Engineering Radio Array (AERA) detects the radio emission in the $30-80 \mathrm{MHz}$ frequency band from extensive air showers with energies larger than $10^{17}$ $\mathrm{eV}$. It consists of more than 150 autonomous radio stations covering an area of about 17 $\mathrm{km}^{2}$. From the distribution of signals measured by the antennas, it is possible to estimate $X_{\max }$. In this contribution three independent methods for the estimation of $X_{\max }$ will be presented.
\end{abstract}

\section{Introduction}

The dominant contribution to the radio emission from extensive air shower is the geomagnetic radiation. It is produced by the deflection of electrons and positrons in the Earth's magnetic field (B) and it is polarized in the direction of the Lorentz force $(\mathbf{v} \times \mathbf{B}$, where $\mathbf{v}$ is the shower axis). A second order contribution comes from a negative charge excess in the shower front: it is called charge excess or Askaryan effect and its radiation is radially polarized towards the shower axis.

The superposition of the geomagnetic and charge-excess radiation results in an asymmetric energy density footprint in the shower plane $(\mathbf{v} \times \mathbf{B}, \mathbf{v} \times \mathbf{v} \times \mathbf{B})$.

The shower observable related to the mass of the primary particle is the atmospheric depth where the shower reaches the maximum number of charged particles $\left(X_{\max }\right)$. Several features of the radio signal are used to estimate $X_{\max }$ : the energy density distribution at ground level; the shape of the frequency spectrum of each individual antenna; the arrival time distribution of the signal at each antenna.

In the following sections the three different methods are described.

\section{$2 X_{\max }$ from the radio energy distribution in the shower plane}

Two different parameterization functions for the radio energy density distribution have been tested on CoReas simulations [1] and AERA datasets.

$\star$ e-mail: canfora@science.ru.nl

${ }^{\star \star}$ Full author list: http://www.auger.org/archive/authors_2018_06.html 

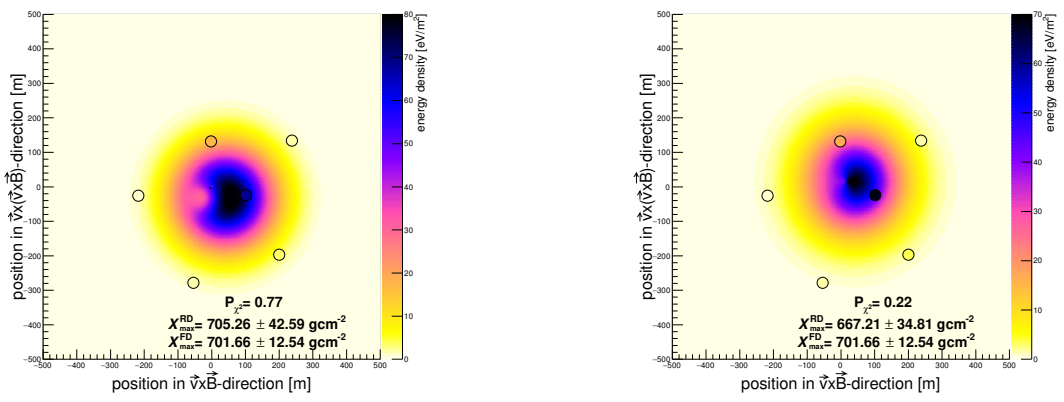

Figure 1. Distribution of the radio signal for an AERA event. The background color map shows the values of the function that is fitted to the $d$ ata: subtraction of two Gaussian distributions (left) and emission mechanisms parameterization (right). In the two plots it is visible the radial asymmetry created by the superposition of the two emission mechanisms. The circles represent the antennas, the color inside the station is the detected energy density. When the color inside the circles matches the background the fit is successful. In both cases the resulting $X_{\max }^{\mathrm{RD}}$ is compatible with the corresponding fluorescence telescopes measurement $\left(X_{\max }^{\mathrm{FD}}\right)$.

The asymmetric energy density footprint can be described analytically by an empirical function consisting of the subtraction of two two-dimensional Gaussian distributions [2]. A linear correlation between the width of the footprint and the geometrical distance to $X_{\max }$ has been found using CoReas iron and proton simulations with zenith angles up to $55^{\circ}$. The measured noise is included in the simulation along with the full detector response. The uncertainty on the reconstruction is obtained by comparing $X_{\max }$ to the corresponding Monte Carlo value and is found to be is $51 \mathrm{gcm}^{-2}$.

The second model, detailed in Ref. [3], uses an analytical description of the geomagnetic and of the charge excess radiation. The parameters of the function are related to physics quantities, such as the radius and the width of the Cherenkov ring which are correlated with the distance to $X_{\max }$. The uncertainty on the $X_{\max }$ reconstruction obtained using the same CoReas simulation sample as before improves to $41 \mathrm{gcm}^{-2}$.

Fig. (1) shows an example of the fit to the energy density for an AERA event. The fit function used in the plot on the left is the subtraction of the two Gaussian distributions, while on the right the geomagnetic and charge-excess parameterization is used. The reconstructed $X_{\max }^{\mathrm{RD}}$ are compatible with the $X_{\max }^{\mathrm{FD}}$ measured using the Auger fluorescence telescopes (FD).

Another method was developed in Ref. [4] and later adapted to AERA data. $X_{\max }$ is obtained by evaluating the agreement of measured radio footprint to air-shower simulations of different $X_{\max }[5,6]$.

\section{$3 X_{\max }$ from spectral information}

A cosmic ray with a lighter mass produces a longer radio pulse compared to heavier primary particles. In the frequency domain a longer pulse results in a steeper spectrum. Therefore the spectral index $(b)$ of the frequency spectrum contains information on $X_{\max }$. Using simulations, $b$ has been parameterized as a function of the arrival direction, the distance antenna-shower axis and the distance to $X_{\max }$ [7]. The parameterization is valid for zenith angles up to $60^{\circ}$ and in the coherent region of the shower outside the Cherenkov cone. Fig. (2) on the left shows a comparison between the spectral index $b$ as function of the distance antenna-shower axis predicted using $X_{\max }$ measured by the fluorescence 

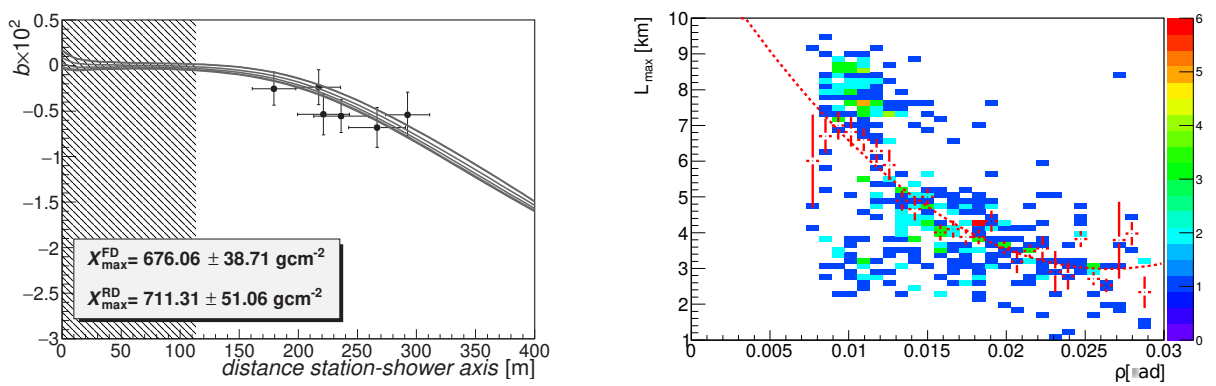

Figure 2. Left: Spectral index $b$ as a function of the distance antenna-shower axis for an AERA event with 6 signal stations. The grey lines are the best predictions obtained using $X_{\max }$ measured by the FD. In the hatched areas the parametrization of the spectral index is not valid. Right: Distance $L_{\max }$ to the shower maximum as a function of the cone opening angle $\rho$ for the simulated dataset. The dashed line is the polynomial function fitted on the profile distribution [9].

telescopes and the spectral index measured by each radio antenna. The radio measurement of each antenna is in agreement with the FD prediction. This method proves that even a single antenna is sensitive to $X_{\max }$.

\section{$4 X_{\max }$ from the arrival time distribution}

The radio wavefront is well described by a hyperboloid. For distances to the shower axis larger than $50 \mathrm{~m}$, the hyperbola can be approximated with a cone and the cone opening angle $(\rho)$ is correlated to the geometrical distance to $X_{\max }\left(L_{\max }\right)$ [8]. Fig. (2) on the right shows $L_{\max }$ as a function of the cone opening angle $\rho$ for a simulated dataset. The study has been performed using proton primaries with energies ranging from $0.28 \mathrm{EeV}$ to $1 \mathrm{EeV}$ and zenith angles up to $50^{\circ}$.

\section{Conclusions}

Three independent techniques for $X_{\max }$ reconstruction based on the radio signal features are currently studied by the Auger collaboration and show promising results. The results of these analyses could be combined to obtain a mass composition reconstruction that uses all the information in the detected radio signal providing a promising technique for the measurement of $X_{\max }$.

\section{References}

[1] T. Huege, M. Ludwig, C.W. James, AIP Conf. Proc. 1535, 128 (2013)

[2] A. Nelles, et al., Astroparticle Physics 60, 13 (2015)

[3] C. Glaser, S. de Jong, M. Erdmann, J.R. Hörandel, arXiv:1806.03620 [astro-ph.HE] (2018)

[4] S. Buitink, et al., Phys. Rev. D90, 082003 (2014)

[5] J. Schulz, Ph.D. thesis, Radboud University Nijmegen (2016)

[6] F. Gaté, for the Pierre Auger Collaboration, in XXV ECRS Conference Proceeding (2016)

[7] S. Jansen, Ph.D. thesis, Radboud University Nijmegen (2016)

[8] W. D. Apel, et al. [LOPES Coll.], Journal of Cosm. and Astrop. Phys. 2014, 025 (2014)

[9] Q.D. Hasankiadeh, for the Pierre Auger Collaboration, in ARENA Conference Proceeding (2014) 